\title{
OBJECTIVE PRISM STUDY OF STAR FORMING REGIONS
}

\author{
M. KUN \\ Konkoly Observatory \\ H-1525 Budapest, Box 67 \\ Hungary
}

Radio molecular observations in the millimeter wavelength region in the last decade have revealed a number of giant molecular cloud complexes at relatively high galactic latitudes. Examples for such cloud complexes are Cepheus Flare (Lebrun 1986), and Ursa Major and Camelopardalis clouds (Heithausen et al. 1993). Because of their high galactic latitudes, these cloud complexes probably belong to the nearest molecular clouds and among them we may find some nearby regions of low-mass star formation.

A study of low-mass star formation in molecular clouds begins with locating the young stellar or protostellar objects within the large area covered by the clouds. Such a task requires widefield radio, submillimeter, infrared and optical observations, taking into account dense molecular cores, outflows, cold embedded objects and optically visible pre-main-sequence stars as relevant objects.

Using the $60 / 90 \mathrm{~cm}$ Schmidt telescope of Konkoly Observatory we have been carrying out an objective prism search for optically visible $\mathrm{H} \alpha$ emission stars in regions of some northern molecular clouds. Our aim is to identify $T$ Tauri star candidates associated with the clouds.

Here, I present some results obtained for the Cepheus Flare molecular cloud complex. Combining them with existing molecular observations and with the IRAS database, new centres of low-mass star formation can be found.

The Cepheus Flare giant molecular cloud complex at a probable average distance of $400 \mathrm{pc}$ contains a mass of about $2 \times 10^{5}$ solar masses (Lebrun 1986). Most of its molecular material is contained in small dark clouds (Lynds 1962). Star formation studies in the region of the Cepheus Flare have been restricted to a few small areas. Particularly scarce information is available on the pre-main-sequence stellar population of the region. The Third Catalog of Emission-Line Stars of the Orion Population (Herbig \& Bell 1988) contains only ten objects in the Cepheus Flare. H $\alpha$ Schmidt surveys have been carried out in L1228 (Ogura \& Sato 1990) and L1251 (Kun \& Prusti 1993).

In order to search for candidate pre-main-sequence stars throughout the Cepheus Flare, we began an $\mathrm{H} \alpha$ survey in 1986 using the $60 / 90 \mathrm{~cm}$ Schmidt telescope of Konkoly Observatory. Kodak 098-02 and Kodak 103aF plates were used in conjunction with an RG1 filter. To date, nearly the whole area of the cloud complex has been covered.

Until now, $130 \mathrm{H} \alpha$ emission stars have been found by visually inspecting the plates. There are a number of previously known emission objects among them, as our survey included the fields 
covered by earlier studies.

The distribution of the $\mathrm{H} \alpha$ emission stars overlaid on the 100 micron IRAS image of the region is displayed in Fig. 1. Several groups of these objects can be recognized. If we believe them to be solar-type pre-main-sequence stars, these groups mark the probable regions of lowmass star formation within the cloud complex.

In addition to the known star-forming regions L1172/NGC7023, L1228 and L1251, high surface density of $\mathrm{H} \alpha$ emission stars are associated with the following clouds: L1199/CB234, L1217/1219, L1235 and L1262.

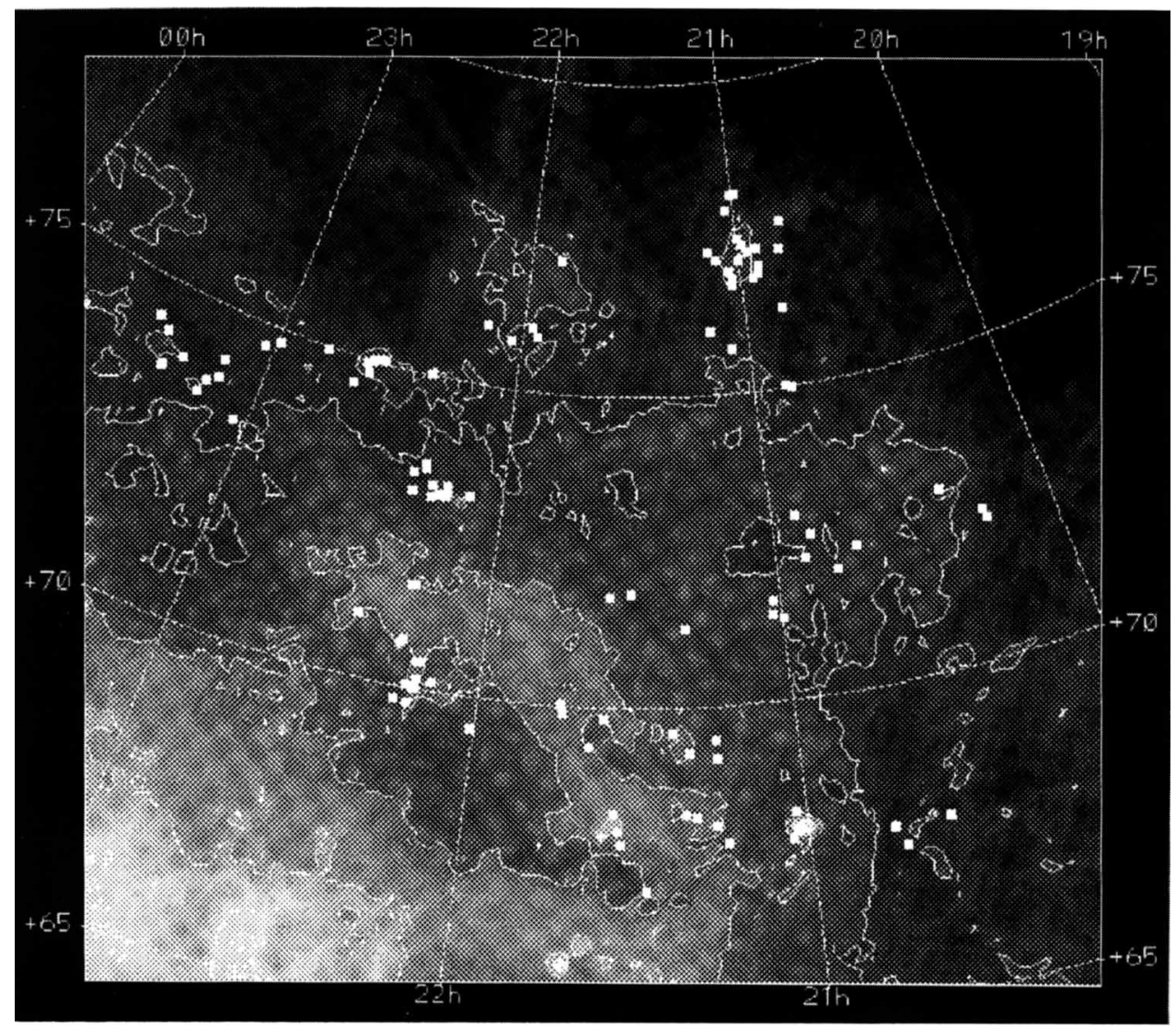

Figure 1. Distribution of the H $\alpha$ emission stars on the 100 micron IRAS image of the Cepheus Flare molecular cloud complex.

\section{Acknowledgements}

This research has been supported by OTKA No. T4341. My participation in the Symposium was financed by a PHARE ACCORD grant through OMFB. 


\section{References}

Heithausen, A., Stacy, J.G., de Vries, H.W., Mebold, U. and Thaddeus, P., 1993. Astron. Astrophys., 268, 265.

Herbig, G.H. and Bell, K.R., 1988. Lick Obs. Bull. No. 1111.

Kun, M. and Prusti, T., 1993. Astron. Astrophys., 272, 235.

Lynds, B.T., 1962. Astrophys. J. Suppl., 7, 1.

Ogura, K. and Sato, F., 1990. Publ. Astron. Soc. Japan, 42, 583. 